\title{
A Diffusion Tensor Imaging Study of Fasciculi in Schizophrenia
}

\author{
Sukhwinder S. Shergill, \\ M.R.C.Psych., Ph.D.
}

Richard A. Kanaan, M.R.C.Psych.

Xavier A. Chitnis, M.Sc.

Owen 0’Daly, M.Sc.

Derek K. Jones, Ph.D.

Sophia Frangou, M.R.C.Psych., Ph.D.

\author{
Steven C.R. Williams, Ph.D. \\ Robert J. Howard, M.R.C.Psych., \\ M.D.
}

\author{
Gareth J. Barker, Ph.D. \\ Robin M. Murray, F.R.C.Psych., \\ D.Sc.
}

\author{
Philip McGuire, F.R.C.Psych., \\ Ph.D.
}
Objective: Cognitive models propose that the symptoms and psychological im- pairments associated with schizophrenia arise as a consequence of impaired com- munication between brain regions, espe- cially the prefrontal cortex and the tem- poral and parietal lobes. Functional imaging and electrophysiological data have provided evidence of functional dys- connectivity, but it is unclear whether this reflects an underlying problem with ana- tomical connectivity. This study used dif- fusion tensor imaging to examine the in- tegrity of the major white matter fasciculi, which connects the frontal and temporal- parietal cortices, and the corpus callosum in patients with schizophrenia.

\begin{abstract}
Method: A 1.5-T magnetic resonance scanner was used to acquire diffusion tensor images giving whole brain coverage at an isotropic $2.5-\mathrm{mm}$ voxel size. Fractional anisotropy was measured in 33 patients with schizophrenia and 40 healthy comparison subjects with an automated voxel-based method of analysis.
\end{abstract}

Results: There was reduced fractional anisotropy in patients with schizophrenia in regions corresponding to the superior longitudinal fasciculi bilaterally and in the genu of the corpus callosum. However, within the patient group, the propensity to experience auditory hallucinations was associated with relatively increased fractional anisotropy in superior longitudinal fasciculi and in the anterior cingulum.

Conclusions: Schizophrenia is associated with altered white matter integrity in the tracts connecting the frontal cortex with the temporal and parietal cortices and with the contralateral frontal and temporal lobes. The severity of these changes may vary with the pattern of symptoms associated with the disorder.
$\mathrm{T}_{\mathrm{b}}$ he pathophysiology of schizophrenia remains unknown, although functional neuroimaging studies have consistently demonstrated the involvement of frontal and temporal cortical regions (1-3). Cognitive models propose that the symptoms of the disorder reflect a disruption of frontal-temporal functional connectivity (4), and there is also evidence of disrupted frontal-temporal and frontalparietal connectivity from functional neuroimaging (5-6), volumetric imaging (7), and electrophysiological investigations (8). Nevertheless, the extent to which these findings reflect differences in the underlying anatomical cortical-cortical connectivity is unclear.

Diffusion tensor imaging data are acquired through the modification of a conventional magnetic resonance imaging (MRI) sequence to permit quantification of the diffusion characteristics of water molecules in vivo (9). Within cerebral white matter, water molecules diffuse more freely along myelinated tracts than across them (10). Such directional dependence of diffusivity is termed "anisotropy," and any reduction in white matter anisotropy indicates a reduction in the degree of tissue order on the voxel scale.
Such structural changes may reflect changes to the underlying white matter tracts, and diffusion tensor imaging can thus be used to examine anatomical connectivity in vivo. However, recent applications of diffusion tensor imaging to schizophrenia have yielded inconsistent results (11-22). Although several studies have reported decreased fractional anisotropy in schizophrenia, there is much less consistency in the topographic location of the white matter changes. This may be related to variations in methodology, both during acquisition (e.g., the extent of brain coverage and the resolution of the images) and during analysis (the relative group size, the selection of the anisotropy measure to be assessed, and whole brain versus region-ofinterest approaches). The corpus callosum has been the most frequently reported site of differences (11-13, 15, 21), although this may be related to the fact that it is the region most frequently examined.

In the present study, we used an optimized diffusion tensor imaging acquisition sequence, permitting whole brain coverage with excellent image resolution, within a time tolerable for patients. We then employed an analysis 
based on automated voxel-based comparisons with conservative-permutation-based statistics to examine changes in fractional anisotropy (one of a number of quantitative measures of anisotropy that can be extracted from the diffusion tensor) in a relatively large group of patients with schizophrenia and matched healthy volunteers. Our first hypothesis, based on the evidence of functional dysconnectivity, was that patients would show reduced fractional anisotropy in the major white matter tracts connecting the frontal cortex with the ipsilateral temporal and parietal cortices (4-8). These tracts include the superior longitudinal fasciculus, which links Wernicke's and Broca's areas, and the uncinate fasciculus, which connects the orbitofrontal and temporal polar cortex. A second prediction, based on data from previous diffusion tensor imaging studies $(11-13,15,21)$, was that there would also be reduced fractional anisotropy in the genu of the corpus callosum. Finally, in view of recent diffusion tensor imaging data linking auditory hallucinations in schizophrenia with changes in increased fractional anisotropy in the arcuate fasciculus and anterior corpus callosum (15), we tested the hypothesis that fractional anisotropy in these regions would correlate with propensity to hallucinations.

\section{Method}

\section{Subjects}

Thirty-nine dextral patients with schizophrenia were scanned. The patients were recruited from wards and clinics at Maudsley Hospital, London. The diagnosis was based on a detailed clinical interview (by S.S.S.) and a review of hospital case notes with DSM-IV criteria (23). In six subjects, the diffusion tensor imaging data were contaminated by significant movement artifacts noted on screening of the images and were not analyzed; this left 30 men and three women with mean age of 32 years $(\mathrm{SD}=10$, range $=$ $18-57)$ and mean premorbid IQ of $108(\mathrm{SD}=8$, range $=94-124)$ that was assessed with the National Adult Reading Test (24). The mean duration of illness was 7 years $(\mathrm{SD}=7$, range $=1-26)$; all but two were being treated with antipsychotic medication at the time of the study.

The patients were assessed with a semistructured interview concerning medical history, including a detailed assessment of auditory hallucinations. The hospital case notes were studied to confirm details of previous clinical presentations. Twenty-six patients had a history of prominent auditory hallucinations and either currently (nine subjects) or during previous episodes of illness ( 17 subjects) had scored 4 or more on items related to hallucinatory behavior on the corresponding item from the Brief Psychiatric Rating Scale (BPRS). Seven subjects had no current auditory hallucinations and had never previously experienced them. Other symptoms, such as formal thought disorder or negative symptoms, were not prominent in the patient group (all patients scored 2 or less on conceptual disorganization and 3 or less on emotional withdrawal and blunted affect items from the BPRS), and there was relatively little variation in the severity of other symptoms across the group. Subsequently, the hallucination subgroups did not differ in the severity of formal thought disorder or negative symptoms. Delusional beliefs were the most common coexisting symptom; two of nine patients in the current hallucinatory group exhibited significant delusions (scoring 4 or more on BPRS items related to suspiciousness and unusual thought content), and the equivalent proportions were 8 of 17 of the previous hallucinating group and two of seven in the group that had never hallucinated. Forty-three healthy subjects, screened to exclude those with any medical/psychiatric disorder, a family history of psychiatric disorder, or any medication, were scanned; diffusion tensor imaging data from three subjects was not analyzed because of significant image artifacts. The group containing 35 men and five women was matched to the patient group for handedness, age (mean $=34$ years, $\mathrm{SD}=9$, range $=19-57$ ), and National Adult Reading Test IQ (mean $=110, \mathrm{SD}=9$, range $=94-$ 123) (Table 1).

For both groups, subjects were excluded if they had a history of head injury, neurological symptoms, or speech or hearing difficulties; fulfilled DSM-IV criteria for abuse or dependence of any illicit drugs or alcohol during their lifetime; or had any contraindications to MRI scanning, including metal implants and claustrophobia. All subjects gave written informed consent, and the study was approved by the local research ethics committee.

\section{Data Acquisition}

Data were acquired with a General Electric Signa LX system (Milwaukee), with actively shielded magnetic field gradients (maximum amplitude $=40 \mathrm{mT} \mathrm{m}^{-1}$ ). A standard quadrature birdcage head coil was used for both radio frequency transmission and magnetic resonance signal reception.

Each diffusion tensor imaging volume was acquired with a multislice, peripherally gated echo planar imaging sequence and optimized (on a healthy volunteer) for precise measurement of the diffusion tensor in parenchyma with over 60 contiguous nearaxial slice locations with isotropic $(2.5 \times 2.5 \times 2.5 \mathrm{~mm})$ voxels. The echo time was $107 \mathrm{msec}$, whereas the effective repetition time was 15 R-R intervals. The duration of the diffusion encoding gradients was $17.3 \mathrm{msec}$, giving a maximum diffusion weighting of 1300 seconds $/ \mathrm{mm}^{2}$. At each slice location, seven images were acquired with no diffusion gradients applied, together with 64 diffusion-weighted images in which gradient directions were uniformly distributed in space. Full details are given elsewhere (25). The diffusion-weighted images were first corrected for an eddycurrent distortion-based registration scheme, as described in Catani et al. (26), and then masked with a modification of the brain extraction tool in the Functional Software Library (FSL) package (Oxford, UK, Oxford University, Centre for Functional MRI of the Brain); see Jones et al. (27) for details. The diffusion tensor was then calculated at each voxel with multivariate linear regression after logarithmic transformation of the signal intensities; fractional anisotropy was calculated at each voxel to produce a multislice fractional anisotropy image (28).

\section{Diffusion Tensor Imaging Processing}

The $b=0$ (non-diffusion-weighted) images from all subjects were mapped to an echo planar imaging template in standard space with a combined affine and low-dimensional nonlinear registration in SPM2 (London, Wellcome Department of Imaging Neuroscience, Functional Imaging Laboratory). This registration aligned all the images and scaled them to the same gross dimensions. Although the $\mathrm{b}=0$ images were $\mathrm{T}_{2}$-weighted, an echo planar imaging template with $\mathrm{T}_{2}{ }^{*}$ weighting was chosen as the initial target rather than a $\mathrm{T}_{2}$-weighted non-echo planar imaging template because the former gives a better match for the distortions in the $b=0$ images. The registered $b=0$ images were then averaged to form a study-specific template to which all $b=0$ images were then reregistered. Images from both patients and comparison subjects were used to make the template so that any registration error would be similar across the two groups. The derived mapping parameters for each subject (from the second registration) were applied to the (inherently coregistered) fractional anisotropy images, which were then thresholded at a value of 0.25 for all 
TABLE 1. Demographic Characteristics of Schizophrenia Patients and Comparison Subjects

\begin{tabular}{|c|c|c|c|c|c|c|c|}
\hline \multirow[t]{2}{*}{$\underline{\text { Variable }}$} & \multicolumn{2}{|c|}{$\begin{array}{l}\text { Schizophrenia Patients } \\
\qquad(\mathrm{N}=33)\end{array}$} & \multicolumn{2}{|c|}{$\begin{array}{c}\text { Comparison Subjects } \\
(\mathrm{N}=40)\end{array}$} & \multirow{2}{*}{$\frac{\text { Analysis }^{\mathrm{a}}}{\mathrm{p}}$} & & \\
\hline & Mean & SD & Mean & SD & & & \\
\hline Age (years) & 32.0 & 10.2 & 34.0 & 8.8 & 0.39 & & \\
\hline \multirow[t]{2}{*}{ IQ } & 108.4 & 7.9 & 110.3 & 6.8 & 0.32 & & \\
\hline & Men & Women & Men & Women & $\mathrm{p}$ & & \\
\hline \multirow{3}{*}{$\begin{array}{l}\text { Gender } \\
\text { Patient subgroups: } \\
\text { hallucination type }\end{array}$} & 30 & 3 & 35 & 5 & 0.64 & & \\
\hline & \multicolumn{2}{|c|}{ Current $(\mathrm{N}=9)$} & \multicolumn{2}{|c|}{ Previous $(N=17)$} & \multicolumn{2}{|c|}{ Never $(N=7)$} & Analysis $^{b}$ \\
\hline & Mean & SD & Mean & SD & Mean & SD & $\mathrm{p}$ \\
\hline Age (years) & 29.9 & 9.7 & 32.8 & 11.3 & 33.1 & 10.2 & 0.78 \\
\hline IQ & 108.3 & 8.2 & 109.5 & 8.1 & 105.7 & 7.8 & 0.63 \\
\hline \multirow[t]{2}{*}{ Illness duration (years) } & 5.7 & 6.3 & 9.1 & 8.0 & 4.3 & 4.5 & 0.19 \\
\hline & Men & Women & Men & Women & Men & Women & $p$ \\
\hline \multirow[t]{2}{*}{ Gender } & 1 & 1 & 17 & 0 & 5 & 2 & 0.09 \\
\hline & Mean & SD & Mean & SD & Mean & SD & $\mathrm{p}$ \\
\hline $\begin{array}{l}\text { Antipsychotic dose in } \\
\text { chlorpromazine } \\
\text { equivalents (mg/day) }\end{array}$ & 388 & 236 & 355 & 237 & 217 & 82 & 0.24 \\
\hline
\end{tabular}

a Mann-Whitney U tests.

${ }^{\mathrm{b}}$ Kruskal-Wallis tests.

subjects, the value empirically found (in a separate investigation) to include all white matter voxels, while minimizing contributions from gray matter or CSF. (Note that while the threshold potentially affects exactly which white matter voxels are included in the analysis, at this lenient value, it only serves to exclude the voxels that are clearly from gray matter or CSF or that lie outside the brain, whose inclusion would otherwise greatly increase the number of statistical comparisons to be made and thus reduce the power of the study.) We applied a 4-mm full-width at halfmaximum smoothing filter to aid between-subject anatomical matching and improve the signal-to-noise ratio. The smoothed registered images were then masked and segmented with Functional Software Library (FMRIB, Oxford, UK), and the white matter segments were used for the subsequent analysis.

\section{Statistical Analysis}

In order to test the null hypothesis, the regression coefficient $\left(\beta_{1}\right)$ of the model $=0$, a test statistic $A\left(\beta_{1} / \mathrm{SE}\left[\beta_{1}\right], \mathrm{SE}=\mathrm{SE}\right)$, was calculated at each voxel. The null distribution was computed by randomly reassigning subjects to two groups of equivalent size to the original data set and performing the same voxelwise test (29). The number of permutations per voxel was 1000 (this number was large enough to permit inference about changes between groups at the voxel level based on the properties of the data at that voxel), with the resulting test statistics forming a distribution under the null hypothesis. This makes the method adaptive to local changes in the properties of the data. The voxelwise statistic image was thresholded at $\mathrm{p}<0.05$, and voxels that were spatially contiguous (in three dimensions) in this thresholded map were assigned to the same cluster. This procedure was repeated as described; the sum of voxel statistics within each cluster was computed for each randomization to form a distribution of cluster mass under the null hypothesis. The mass of each cluster in the observed data was compared to this randomized distribution, and significant clusters were defined as those that had a greater cluster mass than the randomized distribution at a particular significance level. We set the statistical threshold for cluster significance that the expected number of false positive clusters was $<1$ per analysis.

The between-group analysis, as described, was repeated to compare the patients with the healthy comparison subjects and then to examine regions within the patient group that demonstrated changes in propensity to hallucinate by using an analysis of variance (ANOVA) with a hypothesized tendency (currently hallucinating $>$ previously hallucinated $>$ never hallucinated).
The localization of the findings in the white matter was performed with reference to three neuroanatomical atlases (30-32).

\section{Results}

The patients with schizophrenia demonstrated decreased fractional anisotropy, relative to the comparison subjects, within the right frontal and the temporal-parietal portions of both the left and right superior longitudinal fasciculi and in the genu of the corpus callosum. In addition, bilateral decreases in fractional anisotropy were evident in a region encompassing the inferior longitudinal fasiculus and tapetum (Table 2, Figure 1; data supplement Figure 1, available at http://ajp.psychiatryonline.org).

Within the patient group, the propensity to auditory hallucinations was associated with relatively increased fractional anisotropy within the lateral aspects of the superior longitudinal fasciculus bilaterally (Table 2, Figure 2, data supplement Figure 2, Figure 3 in text). The tapetum and anterior cingulum also demonstrated similar relative increases in fractional anisotropy. These areas were distinct from the regions showing decreases in fractional anisotropy in the patients in relation to the comparison subjects; none of these regions demonstrated significantly higher fractional anisotropy values than the comparison subjects (Figure 3) within any of the patient subgroups.

\section{Discussion}

Despite being associated with marked symptoms and cognitive dysfunction, schizophrenia is associated with relatively subtle neuropathological changes in cortical gray matter (33). The absence of marked gray matter changes, as well as recent evidence from functional neuroimaging and electrophysiological studies $(2,4,8)$, is consistent with cognitive models that propose that schizophrenia also involves a disturbance in the connections be- 
TABLE 2. Center of Mass of Significant Changes in Fractional Anisotropy in Schizophrenia Patients and Comparison Subjects

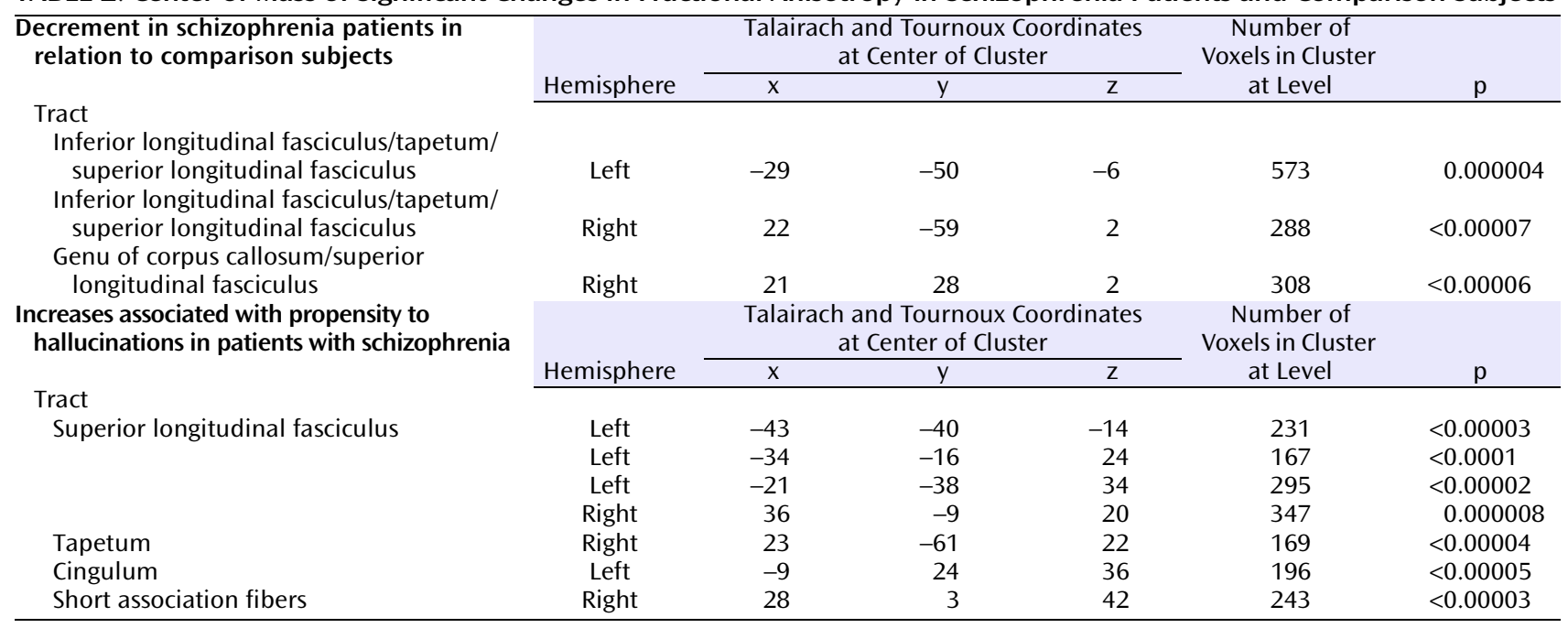

tween gray matter areas. In the present study, we used diffusion tensor imaging in conjunction with a voxel-based analytical approach to examine white matter architecture in schizophrenia. Because it is automated, it reduces the risk of errors due to user intervention and avoids the inherent difficulty in placing regions of interest. We applied this to a group of patients that is larger than has previously been studied with diffusion tensor imaging.

As predicted, we found reduced fractional anisotropy in the superior longitudinal fasciculi bilaterally, consistent with previous diffusion tensor imaging data (15). The superior longitudinal fasciculus is a major association tract connecting large parts of the frontal association cortices with parietal and temporal association areas (30). It forms the main connection between Wernicke's and Broca's areas, which is of particular interest because abnormal language processing (manifest clinically as formal thought disorder and auditory verbal hallucinations) is a key feature of schizophrenia. Contrary to our hypothesis, we did not find reduced fractional anisotropy in the uncinate fasciculus, the other major tract connecting frontal and (anterior) temporal regions, or the cingulum, both of which have shown differences in previous studies $(17,18,20-21)$. This may have been secondary to differences either in patient samples or in the methods used during image acquisition and subsequent analysis, particularly the use of voxelbased methods that allow whole brain testing but may be less powerful than discrete region-of-interest approaches.

Within the patient group, proneness to auditory hallucinations was associated with increased fractional anisotropy within an inferior temporal region of the left superior longitudinal fasciculus and a more superior region bilaterally. However, even in the patients who were most prone to hallucinations, the fractional anisotropy in this region was still lower than in healthy comparison subjects. These findings are broadly consistent with those from a recent study that reported relatively increased fractional anisotropy in a similar part of the superior longitudinal fascicu- lus (the coordinates of their region of interest were at -42 , $-38,4$, compared with $-43,-40,-14$ in this study) with vulnerability to hallucinations, although they reported fractional anisotropy differences that were also significantly greater than their healthy comparison group (15). Although fractional anisotropy changes can be caused by a variety of factors (28), a relative increase in fractional anisotropy within these frontotemporal tracts, especially within these more lateral aspects, could reflect greater connectivity between the lateral frontal and temporal cortex, which might perturb normal communication between areas involved in the generation and monitoring of inner speech $(6,34)$ and contribute to the abnormal coactivation of these regions in functional imaging studies of patients with hallucinations (1-2). However, it is also possible that changes in connectivity may occur as a secondary phenomenon to experiencing auditory hallucinations, with the connections enhanced with the frequency of the hallucinatory experience. This issue could be examined in longitudinal studies of patients with hallucinations, ideally with groups of matched nonhallucinating patients.

The most consistent abnormality in previous diffusion tensor imaging studies of schizophrenia has been reduced fractional anisotropy in the corpus callosum (11$13,15,18,21$ ), and we also found reduced fractional anisotropy in this region, within its anterior portion (the genu). Previous diffusion tensor imaging findings in the corpus callosum have mainly been localized to either its posterior portion $(11,18)$ or to both its anterior and posterior portions. The present findings are compatible with previous reports of decreased volume of the genu in schizophrenia (35) and evidence of functional dysconnectivity between the frontal cortex and contralateral temporal and parietal cortex $(4-6,34)$.

It was difficult to precisely localize the decreased white matter integrity within the region close to the junction of the tapetum and the inferior longitudinal fasciculus. However, based on a diffusion tensor imaging study that de- 
FIGURE 1. Ascending Transverse Sections Through the Brain at Different Levels Relative to the Intercommissural Plane $(\mathrm{mm})^{\mathrm{a}}$

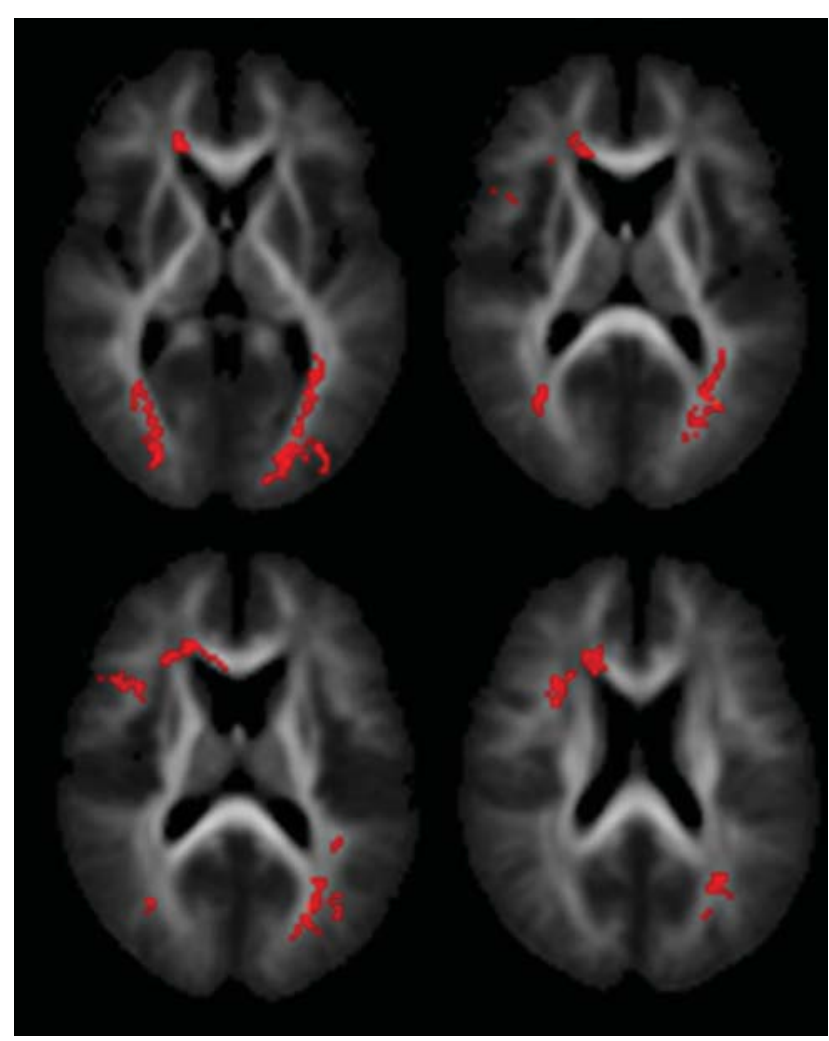

a The right side of the brain is shown on the left side of each section. Regions of white matter demonstrating decreased fractional anisotropy in patients with schizophrenia in relation to comparison subjects are shown in red.

marcated the inferior longitudinal fasciculus with tractography (36), the main area of decreased anisotropy in the present study appears to lie within the tapetum. This is part of the splenium of the corpus callosum connecting homologous regions of the temporal lobes.

Most of the patients were taking psychotropic medication at the time of scanning. Antipsychotic medication has been suggested to influence fractional anisotropy, with one laboratory reporting increased fractional anisotropy with increased antipsychotic dose $(19,20)$. Therefore, we cannot exclude the possibility that differences in fractional anisotropy between patients and comparison subjects were related to medication; however, similar differences have been described in first-episode patients who had received only a few days of antipsychotics and in individuals at high risk of psychosis who were medication naive (37). Similarly, the differences between subgroups of hallucinators were unlikely to be secondary to antipsychotic treatment because neither the current dosage nor the cumulative dosage - as indexed by illness durationdiffered significantly between these subgroups (Table 1). Although age has an effect on anisotropy, this was included as a covariate within the analysis. In view of evi-
FIGURE 2. Ascending Transverse Sections Through the Brain at Different Levels Relative to the Intercommissural Plane $(\mathrm{mm})^{\mathrm{a}}$

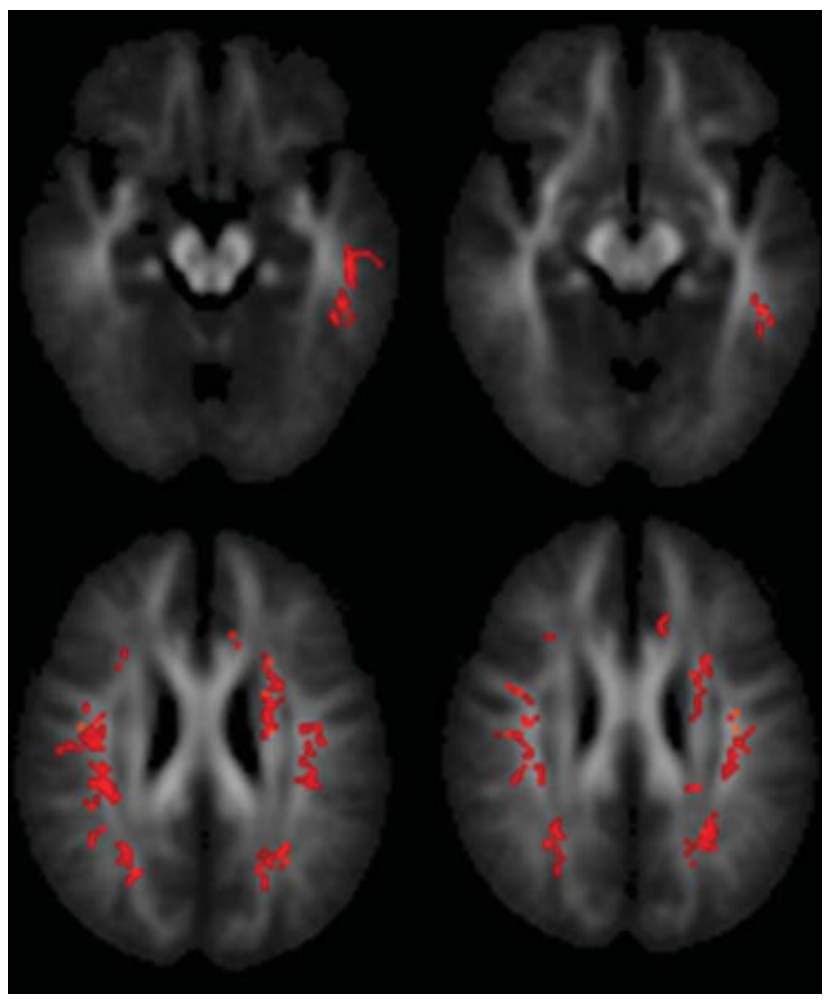

a The right side of the brain is shown on the left side of each section. Regions of white matter demonstrating increased fractional anisotropy in line with propensity to hallucinations are shown in red.

dence that there are progressive volumetric changes in schizophrenia (38), duration of illness may also affect fractional anisotropy. However, in the present study, length of illness was highly correlated with age (Pearson's $r>0.84$ ), so covarying for age is likely to also have eliminated any effects of illness duration. Because of the limited resolution of the diffusion tensor imaging data (2.5-mm voxels), spatial localization errors may be introduced by the registration of data into standard space, and specific tracts were identified through cross-referencing to anatomical atlases. As a result, the localization of the findings to specific tracts is indicative rather than definitive. Diffusion tensor imaging data are exquisitely sensitive to movement artifacts, and it is difficult to exclude any contribution because of systematic differences between groups. An experienced image analyst assessed all the scans while blind to diagnosis and rejected those with movement artifacts. As expected, we had to reject a larger number of patients than comparison scans ( $15 \%$ versus $7 \%$ ).

We thresholded the registered fractional anisotropy images to remove gray matter and CSF voxels and thereby minimize edge effects. Although the same threshold was applied to all images, other operator-independent procedures, such as segmenting the $b=0$ images or a coregistered $\mathrm{T}_{1}$-weighting, may have advantages in terms of iden- 
FIGURE 3. A Scatterplot of the Mean Fractional Anisotropy Values Over the Cluster Located at Talairach Coordinates $(-43,-40,-14)$ Within the Comparison Group $(N=40)$ and Subgroups of the Patient Group

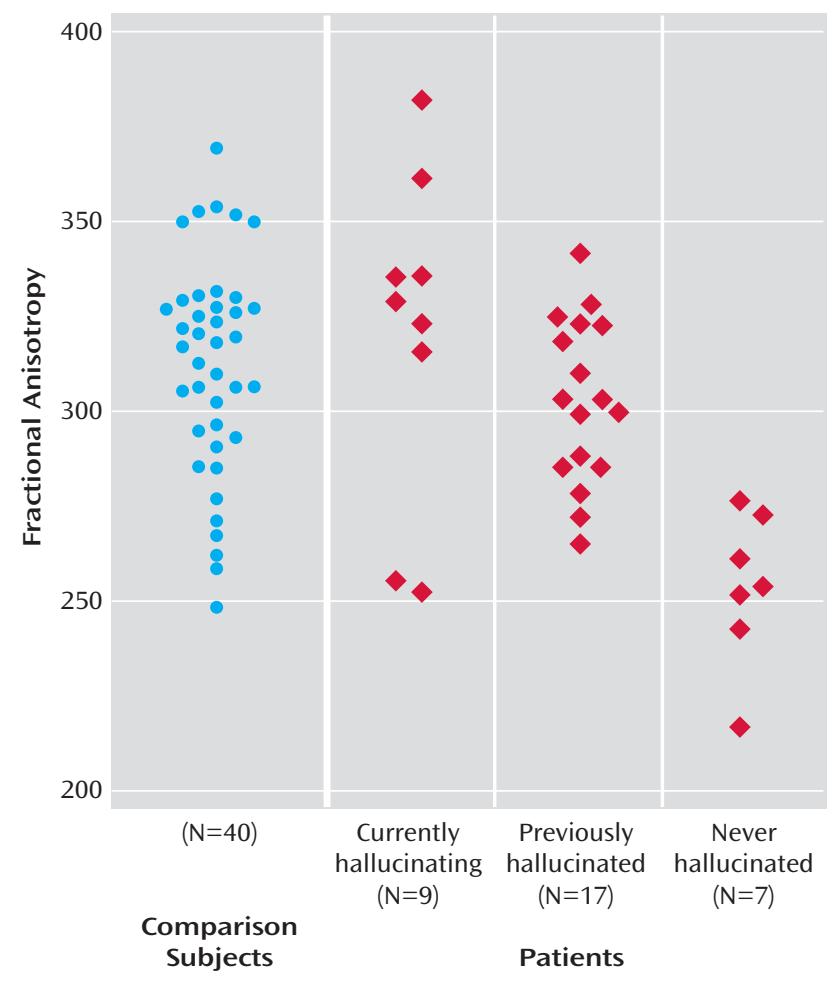

tifying white matter. We used a combined affine and lowdimensional nonlinear registration to map images into standard space. Most previous voxel-based studies of diffusion tensor imaging measures have used either an affine (14) or a similar combination of affine and low-dimensional nonlinear registration (11). Such registration algorithms do not produce exact spatial homology across individuals, and we used spatial smoothing to minimize any error. The use of smoothing inherently sensitizes the analysis to differences with a similar spatial extent to the smoothing kernel used; with no a priori hypothesis as to what size of change to expect, we chose to use only a very small degree of smoothing. This increases the risk of detecting changes that are due to misregistration rather than pathology, so we have replicated the findings in the anterior corpus callosum with a region-of-interest method (39), indicating that at least in this region, the differences in fractional anisotropy were not an artifact of using a voxel-based approach.

In summary, the present study provides evidence of anatomical changes in the tracts connecting the frontal cortex with the temporal and parietal cortices and with the contralateral frontal and temporal lobes in patients with schizophrenia. These changes could underlie the functional dysconnectivity between these regions that has been previously reported in schizophrenia. They may also contribute to the symptoms and cognitive deficits associated with the disorder by interfering with normal mechanisms of motor and cognitive control that rely on rapid communication between distributed cortical regions $(4,40)$.

Received Jan. 16, 2006; revision received March 21, 2006; accepted May 31, 2006. From the Institute of Psychiatry, Kings College London. Address correspondence and reprint requests to Dr. Shergill, Institute of Psychiatry, Kings College London, CSI Lab, P.O. Box 67, De Crespigny Park, London SE5 8AF, U.K.; s.shergill@iop.kcl.ac.uk (e-mail).

All authors report no competing interests.

Dr. Shergill was supported by a Wellcome Advanced Clinical Training fellowship, Dr. Kanaan was supported by an Entry-Level Training Fellowship, Dr. O'Daly was supported by the Psychiatry Research Trust, and Dr. Jones was supported by the Visiting Fellowship Program at NIH and a Wellcome Advanced Training Fellowship.

The authors thank Mark Horsfield, University of Leicester, UK, for his early input in the development of postprocessing software and the Wellcome Trust, who supported this work; and all participating patients and radiographers at the Maudsley Hospital, especially the late Carl Shew.

\section{References}

1. Dierks T, Linden DE, Jandl M, Formisano E, Goebel R, Lanfermann $\mathrm{H}$, Singer W: Activation of Heschl's gyrus during auditory hallucinations. Neuron 1999; 22:615-621

2. Shergill SS, Brammer MJ, Williams S, Murray RM, McGuire PK: Mapping auditory hallucinations in schizophrenia using functional MRI. Arch Gen Psychiatry 2000; 57:1033-1038

3. Kircher T, Liddle PF, Brammer M, Andrew C, Murray RM, McGuire PK: Neural correlates of formal thought disorder in schizophrenia. Arch Gen Psychiatry 2001; 58:767-774

4. Frith CD, Blakemore S, Wolpert DM: Explaining the symptoms of schizophrenia: abnormalities in the awareness of action. Brain Res Rev 2000; 31:357-363

5. Spence SA, Brooks DJ, Hirsch SR, Liddle PF, Meehan J, Grasby PM: A PET study of voluntary movement in schizophrenic patients experiencing passivity phenomena (delusions of alien control). Brain 1997; 120:1997-2011

6. Shergill SS, Bullmore ET, Simmons A, Murray RM, McGuire PK: The functional anatomy of auditory verbal imagery in patients with auditory hallucinations. Am J Psychiatry 2000; 157:16911693

7. Sigmundsson T, Suckling J, Maier M, Williams S, Bullmore E, Greenwood K, Fukuda R, Ron M, Toone B: Structural abnormalities in frontal, temporal, and limbic regions and interconnecting white matter tracts in schizophrenic patients with prominent negative symptoms. Am J Psychiatry 2001; 158:234-243

8. Ford JM, Mathalon DH, Heinks T, Kalba S, Faustman WO, Roth WT: Neurophysiological evidence of corollary discharge dysfunction in schizophrenia. Am J Psychiatry 2001; 158:20692071

9. Le Bihan D, Breton E: Imagerie de diffusion in vivo par resonance magnetique nucleaire. Cr Acad Sci (Paris) 1985; 301: 1109-1112

10. Moseley ME, Cohen Y, Kucharczyk J, Mintorovitch J, Asgari HS, Wendland MR, Tsuruda J, Norman D: Diffusion-weighted MR imaging of anisotropic water diffusion in cat central nervous system. Radiology 1990; 176:439-445

11. Agartz I, Anderson JL, Skare S: Abnormal brain white matter in schizophrenia: a diffusion tensor imaging study. Neuroreport 2001; 12:2251-2254

12. Ardekani BA, Nierenberg J, Hoptman MJ, Javitt DC, Lim KO: MRI study of white matter diffusion anisotropy in schizophrenia. Neuroreport 2003; 14:2025-2029

13. Foong J, Maier M, Clark CA, Barker GJ, Miller DH, Ron MA: Neuropathological abnormalities of corpus callosum in schizo- 
phrenia: a diffusion tensor imaging study. J Neurol Neurosurg Psychiatry 2000; 68:242-244

14. Foong J, Symms MR, Barker GJ, Maier M, Miller DH, Ron MA: Investigating regional white matter in schizophrenia using diffusion tensor imaging. Neuroreport 2002; 13:333-336

15. Hubl D, Koenig T, Strik W, Federspiel A, Kreis R, Boesch C, Maier SE, Schroth G, Lovblad K, Dierks T: Pathways that make voices: white matter changes in auditory hallucinations. Arch Gen Psychiatry 2004; 61:658-668

16. Wang F, Sun Z, Cui L, Du X, Wang X, Zhang H, Cong Z, Hong N, Zhang D: Anterior cingulum abnormalities in male patients with schizophrenia determined through diffusion tensor imaging. Am J Psychiatry 2004; 161:573-575

17. Kubicki M, Westin CF, Nestor PG, Wible CG, Frumin M, Maier SE, Kikinis R, Jolesz FA, McCarley RW, Shenton ME: Cingulate fasciculus integrity disruption in schizophrenia: a magnetic resonance diffusion tensor imaging study. Biol Psychiatry 2003; 54: $1171-1180$

18. Kanaan RA, Kim JS, Kaufmann WE, Pearlson GD, Barker GJ, McGuire PK: Diffusion tensor imaging in schizophrenia. Biol Psychiatry 2005; 58:921-929

19. Okugawa G, Nobuhara K, Sugimoto T, Kinoshita T: Diffusion tensor imaging study of the middle cerebellar peduncles in patients with schizophrenia. Cerebellum 2005; 4:123-127

20. Okugawa G, Nobuhara K, Minami T, Tamagaki C, Takase K, Sugimoto T, Sawada S, Kinoshita T: Subtle disruption of the middle cerebellar peduncles in patients with schizophrenia. Neuropsychobiology 2004; 50:119-123

21. Kubicki M, Park H, Westin CF, Nestor PG, Mulkern RV, Maier SE, Niznikiewicz M, Connor EE, Levitt JJ, Frumin M, Kikinis R, Jolesz FA, McCarley RW, Shenton ME: DTI and MTR abnormalities in schizophrenia: analysis of white matter integrity. Neuroimage 2005; 26:1109-1118

22. Price G, Bagary MS, Cercignani M, Altmann DR, Ron MA: The corpus callosum in first episode schizophrenia: a diffusion tensor imaging study. J Neurol Neurosurg Psychiatry 2005; 76: 585-587

23. American Psychiatric Association: Diagnostic and Statistical Manual of Mental Disorders, 4th ed. Washington, DC, APA, 1994

24. Nelson HE: National Adult Reading Test. Windsor, Berkshire, UK, Nfer-Nelson, 1991

25. Jones DK, Williams SCR, Gasston D, Horsfield MA, Simmons A, Howard R: Isotropic resolution diffusion tensor imaging with whole brain acquisition in a clinically acceptable time. Hum Brain Mapp 2002; 15:216-230
26. Catani M, Howard RJ, Pajevic S, Jones DK: Virtual in vivo interactive dissection of white matter fasciculi in the human brain. Neuroimage 2002; 17:77-94

27. Jones DK, Griffin LD, Alexander DC, Catani M, Horsfield MA, Howard R, Williams SC: Spatial normalization and averaging of diffusion tensor MRI data sets. Neuroimage 2002; 17:592-617

28. Basser PJ, Pierpaoli C: Microstructural and physiological features of tissues elucidated by quantitative-diffusion-tensor MRI. J Magn Reson B 1996; 111:209-219

29. Bullmore ET, Suckling J, Overmeyer S, Rabe-Hesketh S, Taylor E, Brammer MJ: Global, voxel and cluster tests, by theory and permutation, for a difference between two groups of structural MR images of the brain. IEEE Trans Med Image 1999; 18:32-42

30. Dejerine J: Anatomie des Centers Nerveux, vol 1. Paris, Rueff et Cie, 1895

31. Talairach J, Tournoux P: Coplanar Stereotactic Atlas of the Human Brain. Stuttgart, Germany, Thieme, 1988

32. Wakana S, Jiang H, Nagae-Poetscher LM, van Zijl PC, Mori S: Fiber tract-based atlas of human white matter anatomy. Radiology 2004; 230:77-87

33. Harrison PJ: The neuropathology of schizophrenia: a critical review of the data and their interpretation. Brain 1999; 122:593624

34. Shergill SS, Brammer MJ, Fukuda R, Williams SC, Murray RM, McGuire PK: Engagement of brain areas implicated in processing inner speech in people with auditory hallucinations. $\mathrm{Br}$ J Psychiatry 2003; 182:525-531

35. Hulshoff Pol HE, Schnack HG, Mandl RC, Cahn W, Collins DL, Evans AC, Kahn RS: Focal white matter density changes in schizophrenia: reduced interhemispheric connectivity. Neuroimage 2004; 21:27-35

36. Catani M, Jones DK, Donato R, Ffytche DH: Occipito-temporal connections in the human brain. Brain 2003; 126:2093-2107

37. Woolley JB, Barker G, Chitnis X, Broome M, Johns LC, Power P, Wang J, Semple J, Williams SCR, McGuire PK: White matter abnormalities in first episode psychosis and high risk subjects. Schizophr Res 2004; 67:102

38. Pantelis C, Velakoulis D, McGorry PD, Wood SJ, Suckling J, Phillips LJ, Yung AR, Bullmore ET, Brewer W, Soulsby B, Desmond P, McGuire PK: Neuroanatomical abnormalities before and after onset of psychosis: a cross-sectional and longitudinal MRI comparison. Lancet 2003; 361:281-288

39. Kanaan RA, Shergill SS, Barker GJ, Catani M, Ng VW, Howard R, McGuire PK, Jones DK: Tract-specific anisotropy measurements in diffusion tensor imaging. Psychiatry Res 2006; 146:73-82

40. Shergill SS, Bays PM, Frith CD, Wolpert DM: Two eyes for an eye: the neuroscience of force escalation. Science 2003; 301:187 